\title{
Fourth Order Difference Methods for the Initial Boundary-Value Problem for Hyperbolic Equations
}

\author{
By Joseph Oliger
}

\begin{abstract}
Centered difference approximations of fourth order in space and second order in time are applied to the mixed initial boundary-value problem for the hyperbolic equation $u_{t}=-c u_{x}$. A method utilizing third order uncentered differences at the boundaries is shown to be stable and to retain an overall fourth order convergence estimate. Several computational examples illustrate the success of these methods for problems with one and two spacial dimensions. Further examples illustrate the effects of approximations of various orders of accuracy used at the boundaries.
\end{abstract}

1. Introduction. We investigate the problem of obtaining approximate solutions to the initial boundary-value problem for first order hyperbolic equations, e.g.,

$$
\begin{array}{rlrl}
u_{t} & =-c u_{x}, & c>0, a \leqq x \leqq b, t \geqq 0, \\
u(x, 0) & =f(x), & & a \leqq x \leqq b, \\
u(0, t) & =g(t), & & t \geqq 0,
\end{array}
$$

with the compatibility condition $f(0)=g(0)$.

We restrict our attention to finite-difference methods and, further, to those methods which can easily be extended for application to large nonlinear problems in several space dimensions. For this reason, we use second order leap-frog differencing in time $t$ since it is explicit and only requires the storage of two "levels" of the solution. We use the fourth order centered approximation of the first derivative in the spacial coordinate $x$. The mesh ratio should be taken small enough that the $O\left(\Delta t^{2}\right)$ and $O\left(\Delta x^{4}\right)$ error terms are of the same size. The nearly optimal properties of fourth order approximations have been demonstrated in [4] and [9] for the Cauchy problem associated with (1.1). They have been shown to be far superior to lower order approximations and only slightly inferior to sixth order approximations from the standpoint of computational efficiency. Furthermore, little or nothing is gained using even higher order approximations. So long as $c \sim l=b-a$, and certainly for $c<l$, these same conclusions hold for the present problem. The additional complications which arise in the neighborhoods of the boundaries for the problem (1.1)-(1.3) when sixth order methods are used seem to cancel the small advantage in computational efficiency that they have over fourth order methods for the Cauchy problem.

In Section 2, we consider an extrapolation method for handling the closure problem at the boundaries and examine the stability of this technique.

In Section 3, we give several computational results using the extrapolation technique of Section 2 for scalar and vector equations in both one and two space dimen-

Received January 2, 1973.

AMS (MOS) subject classifications (1970). Primary 65M10.

Copyright (c) 1974, American Mathematical Society 
sions. We also compare several other extrapolation procedures with that discussed in Section 2.

We use the theory of Gustafsson, Kreiss, and Sundström [6] and assume that the reader is familiar with that paper.

2. An $O\left(h^{4}\right)$ Approximation with $O\left(h^{3}\right)$ Extrapolation at the Boundaries. We want to compute an approximate solution to the problem (1.1), (1.2), (1.3) using difference methods. For convenience, we take $a=0$ and $b=1$. Let $k>0$ and $h=1 / N, N$ a natural number, and define a grid function $v_{\nu}(t)=v(\nu h, t)$ for $\nu=$ $0,1, \cdots, N$ and $t=0, k, 2 k, \cdots$. For $2 \leqq \nu \leqq N-2$, we approximate (1.1) by the $O\left(h^{4}+k^{2}\right)$ difference approximation

$$
v_{\nu}(t+k)=v_{\nu}(t-k)-c 2 k\left[\frac{4}{3} D_{0}(h)-\frac{1}{3} D_{0}(2 h)\right] v_{\nu}(t)
$$

where $D_{0}(n h) v_{\nu}(t)=(2 n h)^{-1}\left[v_{\nu+n}(t)-v_{\nu-n}(t)\right]$. Let $\lambda=k / h$. For $\nu=1, N-1, N$, we use the following $O\left(h^{3}+k^{2}\right)$ approximations:

$$
\begin{aligned}
& v_{\nu}(t+k)= v_{\nu}(t-k)-c \lambda / 3 \\
& \cdot\left\{-2 v_{\nu-1}(t)-\frac{3}{2}\left[v_{\nu}(t+k)+v_{\nu}(t-k)\right]+6 v_{\nu+1}(t)-v_{\nu+2}(t)\right\} \\
& \text { for } \nu=1,
\end{aligned}
$$

$$
v_{\nu}(t+k)=v_{\nu}(t-k)-c \lambda / 3
$$

$$
\begin{aligned}
& \cdot\left\{v_{\nu-2}(t)-6 v_{\nu-1}(t)+\frac{3}{2}\left[v_{\nu}(t+k)+v_{\nu}(t-k)\right]+2 v_{\nu+1}(t)\right\} \\
v_{\nu}(t+k)= & v_{\nu}(t-k)-c \lambda / 3 \quad \text { for } \nu=N-1, \\
& \cdot\left\{-2 v_{\nu-3}(t)+9 v_{\nu-2}(t)-18 v_{\nu-1}(t)+\frac{11}{2}\left[v_{\nu}(t+k)+v_{\nu}(t-k)\right]\right\} \\
& \text { for } \nu=N .
\end{aligned}
$$

Corresponding to (1.2), we use

$$
v_{\nu}(0)=f(\nu h), \quad \nu=0,1, \cdots, N,
$$

and, corresponding to (1.3), we use

$$
v_{0}(t)=g(t), \quad t=0, k, 2 k, \cdots .
$$

In order to uniquely determine the solution to our difference equations, we assume that we have a sufficiently accurate approximation to the solution $u(x, t)$ at $t=k$, say $w(x)$, and set

$$
v_{\nu}(k)=w(\nu h), \quad \nu=0,1, \cdots, N,
$$

assuming the compatibility relation $w(0)=g(k)$ :

We now investigate the stability of the method defined by (2.1)-(2.7). We use the stability Definition 3.3 of Gustafsson et al. [6]. In [6], it is established (Theorem 5.4) that the stability of two related quarter-plane problems is equivalent to stability for the two-boundary problem for the Definition 3.3. These two problems are simply obtained by removing one or the other of the boundaries and extending the domain to $\pm \infty$, as is appropriate. 
The related right quarter-plane problem consists of Eqs. (2.2), (2.6) and (2.1) for $\nu=2,3, \cdots$ and $(2.5),(2.7)$ for $\nu=0,1,2, \cdots$. For convenience, we replace the related left quarter-plane problem by an equivalent right quarter-plane problem. We consider $c<0$ and use (2.1) for $\nu=2,3, \cdots,(2.7)$ for $\nu=0,1,2, \cdots,(2.5)$,

$$
\begin{aligned}
& v_{\nu}(t+k)= v_{\nu}(t-k)-c \lambda / 3 \\
& \cdot\left\{-\frac{11}{2}\left[v_{\nu}(t+k)+v_{\nu}(t-k)\right]+18 v_{\nu+1}(t)-9 v_{\nu+2}(t)+2 v_{\nu+3}(t)\right\} \\
& \text { for } \nu=0,
\end{aligned}
$$

and (2.2) for $\nu=1$. This completes the definition of the two related problems. We will refer to the first of these as the inflow problem and to the second as the outflow problem.

Hereafter, we always make the

Assumption 2.1. The mesh ratio $\lambda$ is taken to be positive and such that (2.1) is stable for the Cauchy problem, i.e., $0<|c| \lambda<|c| \lambda_{\max } \approx 0.7287$.

In [6], it is shown that stability for the quarter-plane problem is equivalent to certain properties of a determinental equation. We now derive these determinantal equations for our two quarter-plane problems.

Corresponding to Eq. (2.1), there is the resolvent equation

$$
z \hat{v}_{\nu-2}-8 z \hat{v}_{\nu-1}+6 / c \lambda \cdot\left(z^{2}-1\right) \hat{v}_{\nu}+8 z \hat{v}_{\nu+1}-z \hat{v}_{\nu+2}=0
$$

with characteristic equation

$$
P(\kappa)=z-8 z \kappa+6 / c \lambda \cdot\left(z^{2}-1\right) \kappa^{2}+8 z \kappa^{3}-z \kappa^{4} .
$$

Equation (2.8) has resolvent and characteristic equations

$$
\begin{gathered}
3 / c \lambda \cdot\left[(1+11 c \lambda / 6)-(1-11 c \lambda / 6) z^{2}\right] \hat{v}_{\nu} \\
-18 z \hat{v}_{v+1}+9 z \hat{v}_{v+2}-2 z \hat{v}_{v+3}=0, \\
Q_{1}(\kappa)=3 / c \lambda \cdot\left[(1+11 c \lambda / 6)-(1-11 c \lambda / 6) z^{2}\right]-18 z \kappa+9 z \kappa^{2}-2 z \kappa^{3},
\end{gathered}
$$

and for Eq. (2.2) we have the resolvent and characteristic equations

$$
\begin{aligned}
& 2 z \hat{v}_{v-1}+3 / c \lambda \cdot\left[(1+c \lambda / 2)-(1-c \lambda / 2) z^{2}\right] \hat{v}_{v}-6 z \hat{v}_{v+1}+z \hat{v}_{v+2}=0, \\
& Q_{2}(\kappa)=2 z+3 / c \lambda \cdot\left[(1+c \lambda / 2)-(1-c \lambda / 2) z^{2}\right] \kappa-6 z \kappa^{2}+z \kappa^{3} .
\end{aligned}
$$

To continue, we need

LEMMA 2.1. Let $z$ be a complex number. For $|z| \geqq 1$, the roots of Eq. (2.1c) split into two groups $M_{1}=\left[\kappa_{1}(z), \kappa_{2}(z)\right]$ and $M_{2}=\left[\kappa_{3}(z), \kappa_{4}(z)\right]$ such that

(a) $\left|\kappa_{1}(z)\right| \leqq 1,\left|\kappa_{2}(z)\right| \leqq 1$,

(b) $\left|\kappa_{3}(z)\right| \geqq 1,\left|\kappa_{4}(z)\right| \geqq 1$,

where the $\kappa_{i}, i=1, \cdots, 4$, are continuous functions of $z$. Both the inequalities of (a) and of (b) can be taken strictly iff $|z|>1$ or, for $z=e^{i \theta}$ and $\alpha=[6 \sin (\theta) / c \lambda]$, the inequality $\alpha^{2}>9+24 \sqrt{ } 6$ holds. Furthermore, at least one of the inequalities in (a) and in (b) can always be taken strictly.

Proof. This lemma has been proven for $|z|>1$ in [6]. Let $z=e^{i \theta}$. We can then equivalently write $P(\kappa)=0$ as

$$
P_{1}(\kappa)=1-8 \kappa+i(12 / c \lambda) \sin \theta \kappa^{2}+8 \kappa^{3}-\kappa^{4}=0 .
$$


It then is easily verified that $P_{1}(\kappa)=0 \Rightarrow P_{1}\left(\bar{\kappa}^{-1}\right)=0$, i.e., the roots of $P_{1}(\kappa)$ are symmetric in the unit circle. The lemma then follows from a lengthy but straightforward computation based on a theorem of Cohn (see Marden [10, Theorem 45.2]) and Theorem 5.1 of Miller [11].

LEMMA 2.2. The elements of $M_{1}$ are distinct for $|z| \geqq 1$, except for the single value $z_{0}$ which is the root of $z^{2}-c \lambda 6(-36+96 \sqrt{ } 6)^{1 / 2} z-1=0$, such that $\left|z_{0}\right|>1$.

Proof. We can equivalently examine the roots of

$$
P_{2}(\kappa)=1-8 \kappa+\beta \kappa^{2}+8 \kappa^{3}-\kappa^{4}
$$

where $\beta=\left(z^{2}-1\right)(6 c \lambda z)^{-1}$. Let $R\left[P_{2}(\kappa), P_{2}^{\prime}(\kappa)\right]$ be the Bézout resultant of $P_{2}(\kappa)$ and $P_{2}^{\prime}(\kappa)$. It is then necessary that $R$ vanish at multiple roots of $P_{2}(\kappa)$ (Theorem 5 of Collins [1]). We have computed $R$ utilizing the algorithm of Collins ([1], [2]):

$$
R=-\beta^{4}-72 \beta^{2}+54000
$$

which has roots

$$
\begin{array}{ll}
\beta_{1}=(-36+96 \sqrt{ } 6)^{1 / 2}, & \beta_{3}=i(36+96 \sqrt{ } 6)^{1 / 2}, \\
\beta_{2}=-(-36+96 \sqrt{ } 6)^{1 / 2}, & \beta_{4}=-i(36+96 \sqrt{ } 6)^{1 / 2} .
\end{array}
$$

If we then examine the associated roots $\kappa_{i}$ of the quartic $P_{2}$ and the associated roots $z$ of the quadratic defining $\beta$, we find that $\beta_{1}$ yields $\kappa_{1}=\kappa_{2},\left|\kappa_{1}\right|<1 ; \beta_{2}$ yields $\kappa_{3}=\kappa_{4}$, $\left|\kappa_{3}\right|>1, \kappa_{1} \neq \kappa_{2} ; \beta_{3}$ yields $\left|\kappa_{1}\right|<1, \kappa_{2}=\kappa_{3},\left|\kappa_{2}\right|=1,\left|\kappa_{4}\right|>1$ and $\beta_{4}$ yields $\left|\kappa_{1}\right|<1$, $\kappa_{2}=\kappa_{3},\left|\kappa_{2}\right|=1,\left|\kappa_{4}\right|>1$. (Liberty has been taken to number these roots somewhat arbitrarily.) It therefore follows that it is only $\beta_{1}$ which leads to $\kappa_{1}=\kappa_{2}$. The quadratic for $z$ has one root inside and one root outside the unit circle. The lemma follows.

Let $z$ be a fixed complex number, $|z| \geqq 1$, and $\kappa_{1}$ and $\kappa_{2}$ the two roots of (2.1c) belonging to $M_{1}$, on the unit disk. We first consider the case $z \neq z_{0}$ and hence $\kappa_{1} \neq \kappa_{2}$. The general solution for either the inflow or outflow problem is then of the form $\hat{v}_{v}=\rho_{1} \kappa_{1}{ }^{\nu}+\rho_{2} \kappa_{2}{ }^{\nu}$. Then the determinantal equation (see Eq. (10.3) of [6]) associated with our inflow problem is

$$
D_{1}(z) \equiv \operatorname{det}\left(\begin{array}{cc}
1 & 1 \\
Q_{2}\left(\kappa_{1}\right) & Q_{2}\left(\kappa_{2}\right)
\end{array}\right)=Q_{2}\left(\kappa_{2}\right)-Q_{2}\left(\kappa_{1}\right)=0
$$

and that associated with our outflow problem is

$$
D_{2}(z) \equiv \operatorname{det}\left(\begin{array}{ll}
Q_{1}\left(\kappa_{1}\right) & Q_{1}\left(\kappa_{2}\right) \\
Q_{2}\left(\kappa_{1}\right) & Q_{2}\left(\kappa_{2}\right)
\end{array}\right)=Q_{1}\left(\kappa_{1}\right) Q_{2}\left(\kappa_{2}\right)-Q_{1}\left(\kappa_{2}\right) Q_{2}\left(\kappa_{1}\right)=0 .
$$

For $z=z_{0}$, we have $\kappa_{1}=\kappa_{2}$ and thus the general form of the solutions is $\hat{v}_{v}=\left(\rho_{1}+\nu \rho_{2}\right) \kappa_{1}{ }^{\nu}$. Let

$$
\begin{aligned}
& Q_{3}(\kappa)=-18 z \kappa+18 z \kappa^{2}-6 z k^{3} \text { and } \\
& Q_{4}(\kappa)=(3 / c \lambda)\left[(1+c \lambda / 2)-(1-c \lambda / 2) z^{2}\right] \kappa-12 z \kappa^{2}+3 z k^{3} .
\end{aligned}
$$

The determinantal equation associated with the inflow problem is then, at $z=z_{0}$,

$$
D_{1}\left(z_{0}\right) \equiv \operatorname{det}\left(\begin{array}{cc}
1 & 0 \\
Q_{2}\left(\kappa_{1}\right) & Q_{4}\left(\kappa_{1}\right)
\end{array}\right)=Q_{4}\left(\kappa_{1}\right)=0
$$


and that associated with the outflow problem is

$$
D_{2}\left(z_{0}\right) \equiv \operatorname{det}\left(\begin{array}{ll}
Q_{1}\left(\kappa_{1}\right) & Q_{3}\left(\kappa_{1}\right) \\
Q_{2}\left(\kappa_{1}\right) & Q_{4}\left(\kappa_{1}\right)
\end{array}\right)=Q_{1}\left(\kappa_{1}\right) Q_{4}\left(\kappa_{1}\right)-Q_{2}\left(\kappa_{1}\right) Q_{3}\left(\kappa_{1}\right)=0
$$

$D_{1}$ and $D_{2}$ are now defined for all $z$ such that $|z| \geqq 1$.

It is shown in [6] that a necessary and sufficient condition for stability is that the associated determinantal equation has no roots $z$ with $|z| \geqq 1$.

Let $R\left[B_{1}\left(x_{1}, \cdots, x_{n}\right), B_{2}\left(x_{1}, \cdots, x_{n}\right)\right]$ be the Bézout resultant of the polynomials $B_{1}$ and $B_{2}$ in the $n$ complex variables $x_{1}, \cdots, x_{n}$, with respect to the variable $x_{n}$.

Since $\kappa_{1}(z)$ and $\kappa_{2}(z)$ are roots of $P[k(z)]$, we form resultant systems with $P, D_{1}$ and $D_{2} . P, D_{1}$ and $D_{2}$ are polynomials in the variables $\lambda, z, \kappa_{1}$ and $\kappa_{2}$. The resultant system for the inflow problem is

$$
R_{1}(\lambda, z)=R\left\{P\left(\lambda, z, \kappa_{1}\right), R\left[P\left(\lambda, z, \kappa_{2}\right), D_{1}\left(\lambda, z, \kappa_{1}, \kappa_{2}\right)\right]\right\}
$$

and that for the outflow problem is

$$
R_{2}(\lambda, z)=R\left\{P\left(\lambda, z, \kappa_{1}\right), R\left[P\left(\lambda, z, \kappa_{2}\right), D_{2}\left(\lambda, z, \kappa_{1}, \kappa_{2}\right)\right]\right\} .
$$

We can now state

THEOREM (2.1). Let $\lambda$ be fixed. Then, for this $\lambda$, the inflow or outflow problem is stable by Definition 3.3 of [6] if for the associated $R_{i}$ given by (2.11) or (2.12) we have $R_{i}(\lambda, z)=0 \Rightarrow$

(a) $|z|<1$, or

(b) $|z| \geqq 1$ and not both $\kappa_{i}{ }^{*}, i=1,2$, belong to $M_{1}$ where the $\kappa_{i}{ }^{*}$ are the two roots common to $P(z)$ and $D_{i}(z)$.

Proof. Theorem 5 of Collins [1] implies that conditions (a) and (b) imply $D_{i}(z) \neq 0$ for $|z| \geqq 1$. The theorem then follows from Chapter 13 of [6].

The equations $P, D_{1}$ and $D_{2}$ can be written as polynomials with integer coefficients and, consequently, the $R_{i}$ can be computed exactly on a digital computer using, e.g., the algorithm of Collins ([1], [2]). This is quite important since these polynomials are of quite high order and hand computation is out of the question.

We were unable to.verify the conditions (a) and (b) of Theorem 2.1 directly and were forced to verify them computationally. We proceeded in the following manner. First, we computed the roots of the $R_{i}(\lambda)$ obtained using the $D_{i}$ defined by (2.9a) and (2.10a), $z_{i j}(\lambda)$, for fixed $\lambda$. Then, for each root $\left|z_{i j}(\lambda)\right| \geqq 1$, we computed the $\kappa_{i}\left[z_{i}(\lambda)\right], i=1,2$, and then verified the fact that they did not satisfy $D_{i}$ by substitution. For the case $z=z_{0}$, we solved for $\kappa_{1}=\kappa_{2}$ and verified that $R_{i} \neq 0$ by substitution. This process was carried out for a distribution of $\lambda$ values for $0<|c| \lambda<|c| \lambda_{\max }$. The algorithm of Jenkins and Traub [7] was used for all of the root-finding problems.

Within the underlying computational uncertainties of the computational verification of (a) and (b) of Theorem 2.1, we can state

Result 2.1. The difference approximation given by Eqs. (2.1)-(2.7) is stable.

Kreiss [8] has shown that consistent extrapolation techniques at the time level $t$ at the outflow boundary ( $x=b$ in our example) are unstable when used with leap-frog time differencing and the usual $O\left(h^{2}+k^{2}\right)$ leap-frog scheme in the interior. It is easily seen that the same result is also true here. If uncentered, second, third, or fourth order approximations are used instead of (2.3) and (2.4) without the time average at the point $v_{\nu}(t)$ the necessary condition for stability given in Lemma 10.3 
of [6] is violated at the point $z=1$. This is easily seen since the characteristic equation (2.1c) of (2.1) has the root $\kappa=1$ at $z=1$ and the coefficients of the consistent uncentered operators sum to 0 . Therefore, the time averages in (2.3) and (2.4) are essential. We have done test calculations which indicate that the time average in the righthand side of (2.2) used on the inflow boundary is not necessary. However, it is convenient to be able to use the same formula at the point next to the boundary for both inflow and outflow. This is particularly true for certain vector equations when it can reduce the number of operators that need to be coded. For this reason we have not made an extensive study of the use of the formula obtained by eliminating the time average in (2.2).

It should also be noted that we are sacrificing one order of accuracy at the boundaries. Recent convergence results of Gustafsson [5] establish that it is often possible to use approximations of one order lower accuracy on the boundary and still retain the convergence rate of the more accurate interior approximation. The fact that $D_{i}(z) \neq 0$ for $|z| \geqq 1$ fulfills the hypotheses of Gustafsson's Theorem 2.1 [5] and hence establishes that the two quarter-plane problems associated with this method have $O\left(h^{4}\right)$ convergence rates. Our experiments in Section 3 agree with this convergence result. These experiments also show that the use of $O\left(h^{2}\right)$ approximations at the boundary yields significantly larger errors and indicates loss of the $O\left(h^{4}\right)$ convergence rate.

3. Computational Results. In our first set of computations, we compute approximate solutions to the problem (1.1)-(1.3) with $a=0, b=1, c=1, f(x)$, the initial data, defined by

$$
f(x)=\sin (4 \pi x)
$$

and the boundary data

$$
g(t)=f(-t)
$$

This problem has the solution

$$
u(x, t)=f(x-t)
$$

which is 1 -periodic in both $x$ and $t$. Thus, we can equivalently solve (1.1) with initial data (1.2) and replace the boundary condition (1.3) by the periodicity condition

$$
u(0, t)=u(1, t)
$$

We can compute a solution to this periodic boundary problem by approximating (1.1) by (2.1) in the net points $\nu=0,1, \cdots, N-1$, using $v_{N}(t)=v_{0}(t)$, Eq. (2.7), and extrapolating outside the interval $[0,1]$ by the periodicity relation $v_{v}(t)=v_{v+N}(t)$. We should hope that the extrapolation method given by (2.1)-(2.7) would, for small $t$, yield as accurate results as we obtain from the calculation of the equivalent periodic boundary problem. For large $t$, we should expect our extrapolation procedure to yield more accurate results since there should be less accumulated error. We have performed these computations with $N=20, \lambda=\frac{1}{4}$, and using the solution $u\left(x_{v}, k\right)=$ $f(x,-k)=v_{\nu}(k)$ for $(2.7)$. We have chosen $\lambda=\frac{1}{4}$ so that the $O\left(h^{4}\right)$ and $O\left(k^{2}\right)$ error terms are approximately of the same magnitude. The errors in these and subsequent computations of this same problem are given in Table 3.1. This periodic boundary 
TABLE 3.1

\begin{tabular}{lccc|ccc}
\hline Method & $\left\|v_{\nu}\right\|_{2}$ & $\left\|e_{\nu}\right\|_{2}$ & $\left\|e_{\nu}\right\|_{\infty}$ & $\left\|v_{\nu}\right\|_{2}$ & $\left\|e_{\nu}\right\|_{2}$ & $\left\|e_{\nu}\right\|_{\infty}$ \\
\hline & & $t=0.5$ & & & $t=1.0$ & \\
C1 & $7.07-1$ & $4.00-3$ & $5.40-3$ & $7.07-1$ & $8.01-3$ & $1.08-2$ \\
C2 & $7.12-1$ & $9.69-3$ & $2.34-2$ & $7.08-1$ & $1.34-2$ & $2.51-2$ \\
C3 & $7.12-1$ & $1.01-2$ & $2.24-2$ & $7.16-1$ & $1.94-2$ & $4.37-2$ \\
C4 & $6.98-1$ & $8.35-2$ & $1.49-1$ & $6.76-1$ & $1.28-1$ & $2.24-1$ \\
C5 & $6.98-1$ & $2.42-1$ & $4.25-1$ & $6.94-1$ & $3.87-1$ & $8.28-1$ \\
& & $t=2.0$ & & & $t=4.0$ & \\
C1 & $7.07-1$ & $1.60-2$ & $2.16-2$ & $7.07-1$ & $3.20-2$ & $4.32-2$ \\
C2 & $6.96-1$ & $1.30-2$ & $2.04-2$ & $6.96-1$ & $1.25-2$ & $2.28-2$ \\
C3 & $7.14-1$ & $2.73-2$ & $4.18-2$ & $7.13-1$ & $2.72-2$ & $4.33-2$ \\
C4 & $6.25-1$ & $1.88-1$ & $3.31-1$ & $6.16-1$ & $1.75-1$ & $3.02-1$ \\
C5 & $7.33-1$ & $4.44-1$ & $9.68-1$ & $6.90-1$ & $4.80-1$ & 1.05 \\
\hline
\end{tabular}

computation is called $\mathrm{Cl}$ and the computation using the uncentered difference equations (2.2)-(2.4) is called C2. These results confirm our expectations.

We next compare our $O\left(h^{3}+k^{2}\right)$ equations at the boundary with an $O\left(h^{4}+k^{2}\right)$ method. We replace Eqs. (2.2)-(2.4) by

$$
\begin{aligned}
& v_{\nu}(t+k)=v_{\nu}(t-k) \\
& -(\lambda / 12)\left[-6 v_{\nu-1}(t)-20 v_{\nu}(t)+36 v_{\nu+1}(t)-12 v_{\nu+2}(t)+2 v_{\nu+3}(t)\right] \\
& \text { at } \nu=1 \text {, } \\
& v_{\nu}(t+k)=v_{\nu}(t-k) \\
& -(\lambda / 12)\left\{-2 v_{\nu-3}(t)+12 v_{\nu-2}(t)\right. \\
& \left.-36 v_{\nu-1}(t)+10\left[v_{\nu}(t+k)+v_{\nu}(t-k)\right]+6 v_{\nu+1}(t)\right\} \\
& \text { at } \nu=N-1 \text {, } \\
& v_{\nu}(t+k)=v_{\nu}(t-k) \\
& -(\lambda / 12)\left\{6 v_{\nu-4}(t)-32 v_{\nu-3}(t)\right. \\
& \left.+72 v_{\nu-2}(t)-96 v_{\nu-1}(t)+25\left[v_{\nu}(t+k)+v_{\nu}(t-k)\right]\right\} \\
& \text { at } \nu=N \text {. }
\end{aligned}
$$

We call this computation $\mathrm{C} 3$. We only have experimental evidence for the stability of this method. Because of obvious drawbacks, further investigation did not seem worthwhile. We see that there is no loss in accuracy resulting from the use of the $O\left(h^{3}\right)$ equations at the boundaries. It should be noted that Eq. (3.1) does not have $v_{\nu}(t)$ replaced by a time average as in (2.2) but that (3.2) and (3.3) do have $v_{\nu}(t)$ replaced by a time average. This is essential. If $v_{\nu}(t)$ is replaced by $\left[v_{\nu}(t+k)+v_{\nu}(t-k)\right] / 2$ in (3.1), the resulting method is unstable. This has been found to be true by means 
of test computations of the problem now being discussed. We commented earlier on (3.2) and (3.3). Thus, using $O\left(h^{4}\right)$ approximations at the boundary, we must use different operators at the first point inside the boundary for inflow and outflow boundaries. This is contrary to the case where we use $O\left(h^{3}\right)$ approximations and is a definite disadvantage.

We next examine the effect of replacing (2.2)-(2.5) by lower order approximations. We use the $O\left(h^{2}+k^{2}\right)$ approximation

$$
v_{\nu}(t+k)=v_{\nu}(t-k)-\lambda\left[v_{\nu+1}(t)-v_{\nu-1}(t)\right]
$$

for $\nu=1, N-1$ and the $O\left(h+k^{2}\right)$ approximation

$$
v_{\nu}(t+k)=v_{\nu}(t-k)-2 \lambda\left\{\left[v_{\nu}(t+k)+v_{\nu}(t-k)\right] / 2-v_{\nu-1}(t)\right\}
$$

for $\nu=N$. We call this computation C4. The fact that (3.5) is only first order in $h$ instead of second order seems to have no effect, as we have verified by other experiments using $O\left(h^{2}\right)$ extrapolations at the boundary point. We choose to use (3.5), which is due to Arne Sundström, since it seems to be the best extrapolation to use with (3.4).

For comparison purposes, we also include an $O\left(h^{2}+k^{2}\right)$ computation. We use the method due to Sundström and shown to be stable in Elvius and Sundström [3]. This method uses (3.4) for $\nu=1,2, \cdots, N-1$ and (3.5) for $\nu=N$. We call this computation $\mathrm{C} 5$.

Let $e_{\nu}(t)=u\left(x_{\nu}, t\right)-v_{\nu}(t), \nu=0,1, \cdots, N$, be the error at the $\nu$ th grid point. Define norms by

$$
\left\|v_{\nu}(t)\right\|_{2}^{2}=h \sum_{\nu=0}^{N}\left|v_{\nu}(t)\right|^{2} \text { and }\left\|v_{\nu}(t)\right\|_{\infty}=\max \left|v_{\nu}(t)\right| .
$$

In our tables we notate $x \cdot 10^{n}$ by $x+n$. For the initial function we have

$$
\left\|v_{v}(0)\right\|_{2}=0.707 \text {. }
$$

We next test the $O\left(h^{3}\right)$ boundary approximations for a problem with reflective boundary conditions. We use two scalar equations coupled at the boundaries. Let $w(x, t)=[u(x, t), v(x, t)]^{\prime}$. This problem is given by

$$
w_{t}=\left(\begin{array}{rr}
-1 & 0 \\
0 & 1
\end{array}\right) w_{x}, \quad 0 \leqq x \leqq 1, t \geqq 0,
$$

with initial data $w(x, 0)=[f(x), 0]^{\prime}$ where

$$
\begin{aligned}
f(x) & =1-(20 / 3) d, & & \text { if } d<0.15, \\
& =0, & & \text { if } d \geqq 0.15,
\end{aligned}
$$

for $d=\left|x-\frac{1}{2}\right|$ and boundary conditions

$$
u(0, t)=v(0, t), \quad v(1, t)=u(1, t) .
$$

We approximate (3.6) by (2.1) for $2 \leqq \nu \leqq N-2$. We approximate $u_{t}=-u_{x}$ at $\nu=1, N-1, N$ by (2.2), (2.3) and (2.4), respectively. We approximate $v_{t}=v_{x}$ at $\nu=1,2, N-1$ by (2.8), (2.2) and (2.3), respectively. If we denote the solutions of our difference approximation by $W_{\nu}(t)=\left[U_{\nu}(t), V_{\nu}(t)\right]^{\prime}$, the boundary conditions (3.7) 
become

$$
U_{0}(t)=V_{0}(t), \quad V_{N}(t)=U_{N}(t) .
$$

We complete the approximating equations by setting

$$
W_{\nu}(k)=\left(\begin{array}{c}
f\left(x_{\nu}-k\right) \\
0
\end{array}\right) .
$$

The results of this computation are given in Table 3.2. We used $N=.50$ and $\lambda=\frac{1}{4}$. We define

$$
\left|W_{\nu}(t)\right|^{2}=\left|U_{\nu}(t)\right|^{2}+\left|V_{\nu}(t)\right|^{2}
$$

and

$$
\left|e_{\nu}(t)\right|^{2}=\left|u\left(x_{v}, t\right)-U_{\nu}(t)\right|^{2}+\left|v\left(x_{v}, t\right)-V_{\nu}(t)\right|^{2} .
$$

We conclude with two computations in two space dimensions. We have no proof of stability for these methods but stability is indicated. There is evidence that some two-dimensional computations are unstable when methods analogous to those used here are used. In these cases, the algorithms can often be stabilized by adding onedimensional dissipative operators acting on the coordinate tangential to the boundary.

We first consider

$$
w_{t}=\left(\begin{array}{rr}
-1 & 0 \\
0 & 1
\end{array}\right) w_{y}+\left(\begin{array}{rc}
-1 & 0 \\
0 & -1
\end{array}\right) w_{x}, \quad 0 \leqq x \leqq 1,0 \leqq y \leqq 1, t \geqq 0,
$$

where $w(x, y, t)=[u(x, y, t), v(x, y, t)]^{\prime}$. The initial data

$$
w(x, y, 0)=\left(\begin{array}{c}
f(x, y) \\
0
\end{array}\right)
$$

where

$$
\begin{aligned}
f(x, y) & =1-(20 / 3) d, & & \text { if } d<.15, \\
& =0, & & \text { if } d \geqq .15,
\end{aligned}
$$

and $d=\left[\left(x-\frac{1}{2}\right)^{2}+\left(y-\frac{1}{2}\right)^{2}\right]^{1 / 2}$. The boundary conditions are

$$
\begin{array}{ll}
u(x, 0, t)=v(x, 0, t), \quad 0 \leqq x \leqq 1, & u(1, y, t)=u(0, y, t), \quad 0 \leqq y \leqq 1, \\
v(x, 1, t)=v(x, 0, t), \quad 0 \leqq x \leqq 1, & v(1, y, t)=v(0, y, t), \quad 0 \leqq y \leqq 1 .
\end{array}
$$

TABLE 3.2

\begin{tabular}{cccc}
\hline$t$ & $\left\|W_{\nu}(t)\right\| \|_{2}$ & $\left\|e_{\nu}(t)\right\|_{2}$ & $\left\|e_{\nu}(t)\right\| \|_{\infty}$ \\
\hline 0.0 & $3.18-1$ & 0.0 & 0.0 \\
1.0 & $3.18-1$ & $3.40-2$ & $1.04-1$ \\
2.0 & $3.18-1$ & $3.31-2$ & $6.81-2$ \\
3.0 & $3.19-1$ & $3.61-2$ & $8.48-2$ \\
4.0 & $3.20-1$ & $4.93-2$ & $1.24-1$ \\
\hline
\end{tabular}


TABLE 3.3

\begin{tabular}{cccc}
\hline$t$ & $\left\|W_{\nu, \mu}(t) \mid\right\|_{2}$ & $\left\|e_{\nu, \mu}(t)\right\|_{2}$ & $\left\|e_{\nu, \mu}(t)\right\| \|_{\infty}$ \\
\hline 0.0 & $1.09-1$ & 0.0 & 0.0 \\
1.0 & $1.09-1$ & $1.12-2$ & $1.43-1$ \\
2.0 & $1.09-1$ & $1.47-2$ & $1.35-1$ \\
3.0 & $1.09-1$ & $1.88-2$ & $1.29-1$ \\
4.0 & $1.10-1$ & $2.40-2$ & $1.64-1$ \\
\hline
\end{tabular}

TABLE 3.4

\begin{tabular}{cc}
\hline$t$ & $\left\|W_{\nu, \mu}\right\|_{2}$ \\
\hline 0.0 & $1.09-1$ \\
1.0 & $1.11-1$ \\
2.0 & $1.12-1$ \\
3.0 & $1.12-1$ \\
4.0 & $1.13-1$ \\
\hline
\end{tabular}

Thus, we have reflective boundary conditions in the $y$-direction and periodic boundary conditions in the $x$-direction. We use equal grid intervals in the $x$ - and $y$-coordinates, $h=1 / N, N=50$ and take $\lambda=\frac{1}{4}$. We again use an $O\left(h^{4}+k^{2}\right)$ approximation in the interior and $O\left(h^{3}+k^{2}\right)$ approximations near the boundaries $y=0$ and $y=1$. We approximate the $\partial_{y}$ operator here exactly as we approximated the $\partial_{x}$ operator in our last example and the $\partial_{x}$ operator here is approximated with our usual centered $O\left(h^{4}\right)$ approximation and the periodicity condition in the $x$-coordinate in the neighborhoods of the boundaries $x=0$ and $x=1$. We use the solution $w(x, y, k)=$ $[f(x-k, y-k), 0]^{\prime}$ as our approximation at time $t=k$. Results of this computation are given in Table 3.3.

Let $W_{\nu, \mu}(t)=\left[U_{\nu, \mu}(t), V_{\nu, \mu}(t)\right], U_{\nu, \mu}(t)=U(\nu h, \mu h, t), V_{\nu, \mu}(t)=V(\nu h, \mu h, t)$ be the solution to the difference approximation and define $\left|W_{\nu, \mu}\right|^{2}$ and $\left|e_{\nu, \mu}\right|^{2}$ as before. Define norms for a scalar grid function by

$$
\left\|v_{\nu, \mu}\right\|_{2}^{2}=h^{2} \sum_{\nu=1}^{N} \sum_{\mu=1}^{N}\left|v_{\nu, \mu}\right|^{2}, \quad\left\|v_{\nu, \mu}\right\|_{\infty}=\max _{1 \leqq \nu \leqq N ; 1 \leqq \mu \leqq N}\left|v_{\nu, \mu}\right| .
$$

For our last computation, we computed an approximate solution of the equation

$$
w_{\imath}=\left(\begin{array}{cc}
-1 & 0 \\
0 & 1
\end{array}\right) w_{y}+\left(\begin{array}{ll}
0 & 1 \\
1 & 0
\end{array}\right) w_{x}, \quad 0 \leqq x \leqq 1,0 \leqq y \leqq 1, t \geqq 0,
$$

with the same initial and boundary conditions as in the previous example. We used the same grid and analogous difference approximations. We give $\left\|W_{\nu, \mu}\right\|_{2}$ for this computation in Table 3.4 . 
Acknowledgments. This work was begun while the author was at the International Meteorological Institute, Stockholm, Sweden. Preliminary computations were done there on the IBM 360/75 of the University of Stockholm with the programming assistance of Henry Larsson. The computer results given in this paper were obtained on the CDC 6600 and 7600 machines of the National Center for Atmospheric Research, Boulder, Colorado. Richard Helgason assisted with the algebraic manipulation routines.

The author thanks Professor Heinz-Otto Kreiss of the University of Uppsala for many helpful conversations.

National Center for Atmospheric Research

Boulder, Colorado 80302*

1. G. E. Collins, "The calculation of multivariate polynomial resultants," J. Assoc. Comput. Mach., v. 18, 1971, pp. 515-532. MR 45 \#7970.

2. G. E. Collins, The SAC-1 Polynomial GCD and Resultant System, Report 145, Computer Sciences Department, University of Wisconsin, Madison, 1972.

3. T. Elvius \& A. SUNDSTRÖM, "Computationally efficient schemes and boundary conditions for a fine-mesh barotropic model based on the shallow-water equations," Tellus, v. 25,1973 , pp. $132-156$.

4. B. ForNBERG, On High Order Approximations of Hyperbolic Partial Differential Equations by a Fourier Method, Report 39, Department of Computer Sciences, Uppsala University, Uppsala, Sweden, 1972.

5. B. GuSTAFsson, On the Convergence Rate for Difference Approximations to Mixed Initial Boundary Value Problems, Report 33, Department of Computer Sciences, Uppsala University, Uppsala, Sweden, 1971.

6. B. GUSTAFSSON, H.-O. Kreiss \& A. SUNDSTRöM, "Stability theory of difference approximations for mixed initial boundary value problems. II," Math. Comp., v. 26, 1972, pp. $649-686$.

7. M. A. Jenkins \& J. F. Traub, "A three-stage variable-shift iteration for polynomial zeros and its relation to generalized Rayleigh iteration," Numer. Math., v. 14, 1969/70, pp. 252-263. MR 41 \#2918.

8. H.-O. KREISS, "Difference approximations for initial boundary value problems," Proc. Roy. Soc. London Ser. A, v. 323, 1971, pp. 255-261.

9. H.-O. KREISs \& J. Oliger, "Comparison of accurate methods for the integration of hyperbolic equations," Tellus, v. 24, 1972, pp. 199-215.

10. M. MARDEN, Geometry of Polynomials, 2nd ed., Math. Surveys, no. 3, Amer. Math. Soc., Providence, R.I., 1966. MR 37 \#1562.

11. J. J. H. MILler, "On the location of zeros of certain classes of polynomials with applications to numerical analysis," J. Inst. Math. Appl., v. 8, 1971, pp. 397-406. MR 45 \#9481.

* The National Center for Atmospheric Research is sponsored by the National Science Foundation. 